\title{
Comprehensive Multidisciplinary Rehabilitation of Arthrogryposis Multiplex Congenita
}

\author{
Longjam Darendrajit Singh ${ }^{1}$, Akoijam Joy Singh ${ }^{2}$, Longjam Nilachandra Singh ${ }^{2}$ \\ ${ }^{1}$ Department of Physical Medicine and Rehabilitation, All India Institute of Medical Sciences, Bhubaneswar, India; ${ }^{2}$ Department of \\ Physical Medicine and Rehabilitation, Regional Institute of Medical Sciences, Imphal, India.
}

\section{Corresponding Author: \\ Mr. Longjam Darendrajit Singh \\ Email: darendrajit4u@gmail.com}

This is an Open Access article distributed under the terms of the Creative Commons Attribution License (creativecommons.org) licenses/by/3.0).

Received : July 14, 2020

Accepted : August 31,2020

Published : October 10,2020

\begin{abstract}
Background: Arthrogryposis multiplex congenita (AMC) refers to the development of multiple joint contractures affecting two or more areas of the body prior to birth. A contracture occurs when a joint becomes permanently fixed in a bent or straightened position which can impact the function and range of motion of the joint and may lead to muscle atrophy. Rehabilitation of a case of AMC is still a challenge owing to its multiple system involvement. Case Report: The patient presented at the age of 14 years with multiple joint contractures, dependent in most of the ADLs- eating, bathing, toileting and dressing upper garment, independent in transfer, bed mobility and dressing lower garment (FIM score=99). She walked on knees leading to skin hypertrophy though she could stand on one limb. Conclusion: Multidisciplinary approach consisting of a physiatrist, occupational therapist, prosthetist and orthotist, psychotherapist, physical therapist, art therapist, and vocational counselor is essential for the treatment. With extensive comprehensive multidisciplinary rehabilitation program the patient could walk 100 metres with orthoprosthesis, independent in most of the ADLs (FIM score improved from 99 to 115). She expressed great satisfaction on being able to walk upright for the first time in her life.
\end{abstract}

Keywords: Activities of Daily Living, Arthrogryposis, Arthroplasty, Contracture, Knee Prosthesis.

\section{Introduction}

Arthrogryposis multiplex congenita, or simply arthrogryposis is derived from the Greek words arthron - joint, gryposis - curvature, and literally means curved or crooked joints. This is a rare inherited disease that is characterized by multiple configurations of congenital limb contractures, usually non-progressive and often gradually improving with appropriate management [1]. It occurs in two or more areas of the body with an incidence rate of 1 in 3000 live births [2] and is usually a feature of abnormal neurologic development or primary muscular disorders of the fetus. It is not a specific diagnosis but rather is a descriptive term for multiple contractures that can be associated with many different medical conditions. More than 400 conditions are associated with this finding, which makes a specific prenatal diagnosis challenging [3].

The causes of arthrogrypotic syndromes are largely unknown, but are presumed to be multifactorial. Potential etiologies have been grouped by Hall into neuropathic abnormality, abnormality of muscle structure or function, abnormality of connective tissue, space limitation, maternal diseases and impaired intrauterine or fetal vascularity $[3,4]$. Neuropathic abnormality is associated with spinal cord changes, which includes loss of motor neurons resulting in muscle atrophy. Traditionally it has been thought that $95 \%$ of 
arthrogryposis was due to neuropathies, but many myopathic genes have been recently discovered [5-7]. When a joint is not moved for a period of time, extra-connective tissue may grow around it, fixing it in place. Lack of joint movement also means that tendons connected to the joint are not stretched to their normal length, which can make normal joint movement difficult [3]. The common end pathway is lack of fetal joint movement after initially normal development, leading to collagen proliferation, fibrotic replacement of muscle, and a marked thickening of joint capsules (the collagenic response) [8]. Generally AMC is sporadic, however symptoms of some forms of arthrogryposis can be found in selected monogenic diseases (with the autosomal recessive, autosomal dominant or X-linked inheritance), metabolic diseases (e.g. mitochondrial diseases), chromosome aberrations, microdeletion and micro-duplications $[9,10]$. The classical form of AMC, amyoplasia, is usually sporadic. Lack of genetic background seems to be confirmed by the fact that amyoplasia is common in only one of monozygotic twins [11]. Distal arthrogryposes, on the other hand, are a specific subgroup of disorders, which may be inherited in an autosomal dominant pattern, with phenotypic variation between families and even between members of the same family. Distal arthrogryposis syndromes are caused by mutation in several different genes, each of which encodes a component of the contractile apparatus [11].

\section{Case Report}

A 14 year old female attended Physical Medicine and Rehabilitation out-patient department of Regional Institute of Medical Sciences, Imphal, India with complaints of stiffness and deformity of multiple joints since birth. She has history of polyhydramnios in the prenatal period. She had delayed motor milestones. She is the third child of the family and there is no similar disease among the siblings. She was diagnosed as having AMC after birth because of the characteristic appearance and multiple contractures of the limb joints. She was managed conservatively with stretching, range of motion (ROM) exercises and splintings. On examination she was of average built with fair nutrition, $124 \mathrm{~cm}$ in height, weighed $39 \mathrm{~kg}$. She had generalized muscle atrophy, flexion contractures of both upper and lower limb joints, exaggerated lumbar lordosis, pterygium of the neck and elbows. She had generalized restriction in ROM of both upper and lower limb joints [Table 1]. Right knee had a flexion contracture of 60 degrees with fixed equinus deformity of 50 degrees at the right ankle. Left knee had ROM of 30 degrees [Fig.1]. Motor powers were 4 proximally and 5 distally (MRC scale) in both upper and lower limbs. Sensation was intact. Laboratory tests were normal. She was dependent in eating, bathing, toileting and

Table 1: Upper and lower limb joint ROMs.

\begin{tabular}{|c|c|c|c|c|}
\hline Joint & Movement & $\begin{array}{l}\text { Normal } \\
\text { range }\end{array}$ & Right & Left \\
\hline \multirow[t]{4}{*}{ Shoulder } & Flexion & $0-170^{\circ}$ & $140^{\circ}$ & $150^{\circ}$ \\
\hline & Abduction & $0-170^{\circ}$ & $130^{\circ}$ & $140^{\circ}$ \\
\hline & Internal rotation & $0-70^{\circ}$ & $30^{\circ}$ & Nil \\
\hline & External rotation & $0-90^{\circ}$ & $10^{\circ}$ & Nil \\
\hline Elbow & Flexion & $0-140^{\circ}$ & $80^{\circ}$ & $50^{\circ}$ \\
\hline \multirow{2}{*}{$\begin{array}{l}\text { Radio- } \\
\text { ulnar }\end{array}$} & Supination & $0-80^{\circ}$ to $90^{\circ}$ & $35^{\circ}$ & $20^{\circ}$ \\
\hline & Pronation & $0-80$ to $90^{\circ}$ & $70^{\circ}$ & $80^{\circ}$ \\
\hline \multirow[t]{4}{*}{ Wrist } & Flexion & $0-80^{\circ}$ & $40^{\circ}$ & $40^{\circ}$ \\
\hline & Extension & $0-70^{\circ}$ & $20^{\circ}$ & $20^{\circ}$ \\
\hline & Ulnar deviation & $0-30^{\circ}$ & $20^{\circ}$ & $15^{\circ}$ \\
\hline & Radial deviation & $0-12^{\circ}$ & $7^{\circ}$ & $10^{\circ}$ \\
\hline \multirow[t]{4}{*}{ Hip } & Flexion & $0-120^{\circ}$ & $105^{\circ}$ & $110^{\circ}$ \\
\hline & Abduction & $0-40^{\circ}$ & $40^{\circ}$ & $35^{\circ}$ \\
\hline & Internal rotation & $0-45^{\circ}$ & $10^{\circ}$ & - \\
\hline & External rotation & $0-45^{\circ}$ & $35^{\circ}$ & - \\
\hline \multirow[t]{2}{*}{ Knee } & Flexion & $0-135^{\circ}$ & $60^{\circ}-120^{\circ}$ & $30^{\circ}$ \\
\hline & Flexion deformity & - & $60^{\circ}$ & - \\
\hline \multirow[t]{5}{*}{ Ankle } & Plantar flexion & $0-50^{\circ}$ & - & $35^{\circ}$ \\
\hline & Dorsi flexion & $0-15$ & - & $5^{\circ}$ \\
\hline & Equinus deformity & - & $\begin{array}{l}60^{\circ} \\
\text { (Fixed) }\end{array}$ & - \\
\hline & Inversion & $0-35^{\circ}$ & $\begin{array}{l}20^{\circ} \\
\text { (Fixed) }\end{array}$ & $15^{\circ}$ \\
\hline & Eversion & $0-20^{\circ}$ & - & $15^{\circ}$ \\
\hline
\end{tabular}


dressing upper garment, independent in transfer, bed mobility and dressing lower garment (FIM score=99). She walked on knees leading to hypertrophy of the skin. She could stand on one limb (Single limb stance test, eye opened=15 sec, eye closed $=4 \mathrm{sec}$ ).

Patient was admitted in the ward for extensive multidisciplinary rehabilitation program. The main goals of rehabilitation were to improve balance, ambulation, to increase independent function of the upper limbs and to improve the activities of daily living. Stretching and passive ROM exercises for upper and lower limb joint contractures, posture and balance-proprioceptive training and active resistive extremity strengthening were done. Therapeutic ultrasound was given to the contracted muscles before stretching exercises and electrical stimulations were performed to increase the strength of related muscles. To make the patient independent in ADLs various modifications were made like universal cuff with spoon for eating, long handled bath sponges and bath mitt for bathing. Patient was not able to wear upper garments by herself because of the restrictions in the shoulder joints ROM. We devised a simple customized hook using locally available iron rods fixed to a wall for dressing of the upper garments [Fig.2]. Her FIM score improved from 99 to 115 .

Minimal improvements were noted in passive ROM measurements of both shoulders and knees. However patient showed improvements in balance function (Single limb stance test improved from eye opened $=10 \mathrm{sec}$, eye closed $=4$ sec (admission) to eye opened $=26 \mathrm{sec}$, eye closed $=$ $6 \mathrm{sec}$ (discharge). As for the case we proposed soft tissue lengthening surgery with possibility of Ilizarov application for the correction of right knee flexion deformity or transfemoral amputation with prosthesis fitting. But patient's parents didn't give the consent for surgery. An orthoprosthesis (extension prosthesis) was devised to accommodate the flexion deformity of the right knee. It consisted of an anterior opening thigh shell for easy donning

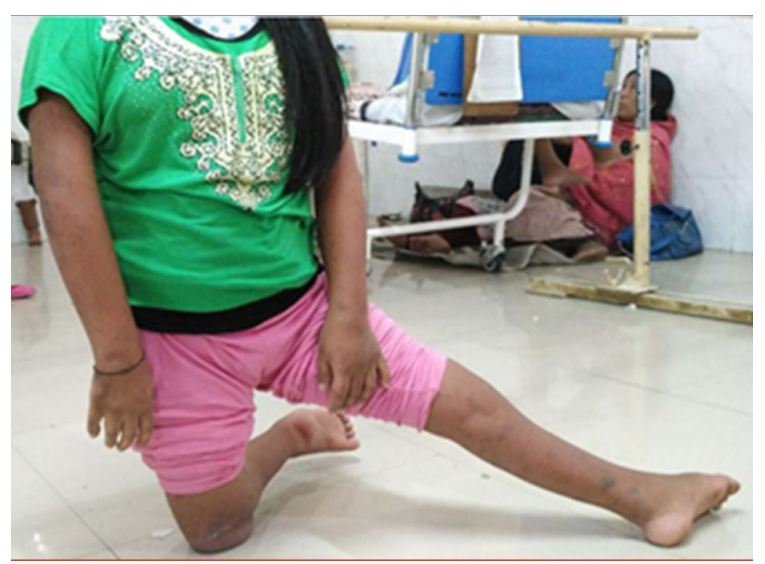

Fig.1: Patient standing on knee (flexion deformity right knee, equinous deformity right ankle).

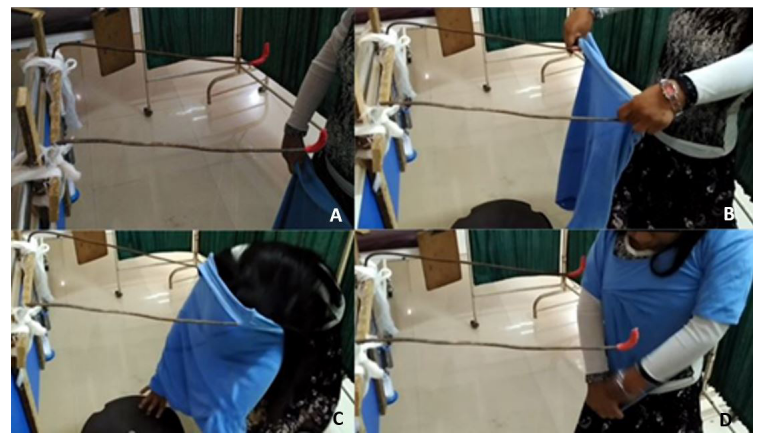

Fig.2: Dressing of the upper garment using customized iron hooks.

and doffing, connected to a calf shell by an external mechanical single axis hinge joints and a SACH foot [Fig.3]. Patient continued extensive rehabilitation program including balance and gait training with the orthoprosthesis for 10 weeks. The patient was discharged after 3 months of extensive rehabilitation program. At the time of discharge she was able to walk about 100 metres, independent in most of the ADLs. She expressed great satisfaction on being able to walk upright for the first time in her life. She was advised to continue rehabilitation program at home.

\section{Discussion}

Arthrogryposis is a symptom rather than a diagnosis. It implies contractures in multiple body areas usually involving the limbs, but may also include limitation of full range of movement 
of the jaw, neck, and spine at birth [3]. Although the etiology of disease is not clear, many genetic defects are shown in the pathogenesis of AMC. These may be due to single-gene mutations, chromosomal abnormalities, and mitochondrial defects [1]. Amyoplasia is a sporadic condition and the intelligence is normal and there is no major malformation in the central nervous system, heart, gastrointestinal tract, and genitourinary system in patient with amyoplasia [12]. Our case was sporadic and history of family was normal and diagnosis had been made just after the birth due to characteristic multiple joint contractures resulting in delayed motor milestones.

Although the main problem is multiple joint contractures in $\mathrm{AMC}$, it may be accompanied by the other symptoms. These can include thin skin, muscle atrophies, limb anomalies (such as shortness, webs, radial head dislocations, and patellar aplasia), abnormalities of face and jaw (asymmetry, depressed nasal bridge, micrognathia, trismus, and hemangioma), scoliosis, and different anomalies of respiratory, urinary, and nervous systems [13]. In this case, patient's symptoms were not only multiple contractures of joints, but also pterygiums on neck and elbows, and muscular hypoplasia. There was no pathology in the other visceral system.

Joint development occurs during embryogenesis in the first 8 weeks of intrauterine life. Impairment of intrauterine movement leads to contractures, and the more severe the restriction in movement is, or the longer it occurs, the worse are the resultant contractures [14,15]. Arthrogryposis should be suspected if prenatal ultrasound examination shows an absence of fetal movement, especially if accompanied by polyhydramnios [16]. At birth, children will have fixed contractures and the limbs are tubular and featureless, lacking the normal skin creases seen around joints. A deep dimple is often seen over joints as a result of the skin being adherent to the underlying bone. The trunk may also be affected and devoid

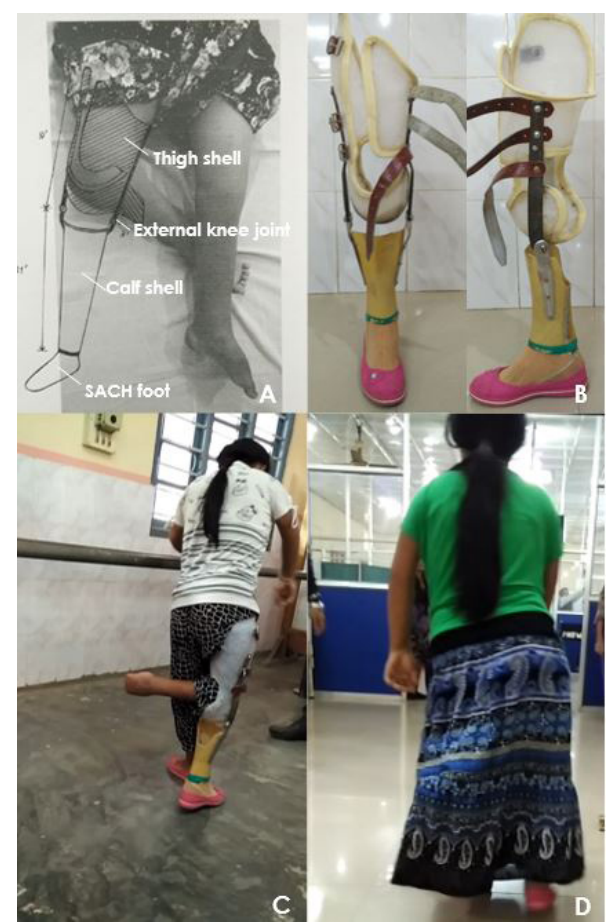

Fig.3: Orthoprosthesis fitment and training.

of creases, giving a characteristic appearance. When the upper limbs are affected, they take a particular attitude: shoulders on internal rotation and adduction, elbows fixed on extension, wrists on palmar flexion and ulnar deviation, thumbs on adduction into the palms, and fingers fixed in a flexed posture. In the lower limbs, the hips are flexed, abducted and externally rotated, congenital dislocation of the hips may also be seen. The most common lower limb deformities are rigid clubfoot and knee contractures, which are more commonly extended, rather than flexed. The limbs are not painful, but there is only a little movement in the affected joints with an inelastic endpoint beyond a limited range of movement [17]. Scoliosis is reported in 10-30\% of cases [3]. The present case had generalized restriction in ROM of both upper and lower limbs, with fixed flexion deformity of 60 degrees at the right knee with a fixed equinus deformityof 50 degrees on right side and flexion range of 30 degrees on the left knee.

Treatment must be planned according to the patient; long-term rehabilitation programs 
at home should be explained to the patient and caregivers. Multidisciplinary approach is essential for the treatment. The team consists of physiatrist, occupational therapist, prosthetist and orthotist, psychotherapist, physical therapist, art therapist, vocational counselor etc. Splints or serial casting, passive stretching, and range of motion exercises are particularly effective in the treatment of contractures [18]. Although patients of the classical arthrogryposis gain back their independence in their activities of daily living some patients may need help from other people even in adulthood [13]. Corrective surgery, focused on addressing lower limb deformity that would prevent standing and walking can be done early. As for the upper limbs, surgery is usually delayed until later in childhood so that adequate assessment of hand function can be made, to allow the surgical intervention to be individualized to the child's needs. However, major joint repositioning surgery should probably be performed before the age of four. With increasing age, there is increasing occurrence of the joint stiffness and limbs abnormal positions.

As for the present case the patient was given the option of soft tissue lengthening surgery with possibility of Ilizarov application, transfemoral amputation of the right lower limb with prosthesis fitment or orthoprosthesis, also called knee bent prosthesis application. Patient's parents refused to undergo any surgery. Customized orthoprosthesis fitment was done. Reintegration tries to compensate for the deficiencies and limitations activities that persist despite rehabilitation, with the aim of allowing the best possible integration of the person in their environment. This process requires some material assistance such as medical devices and technical aids, aids to rehabilitation, human helps, adaptations of the environment in the private or public domain.

\section{Conclusion}

Management in Physical Medicine and Rehabilitation should be based on comprehensive multidisciplinary care with the aim of enabling the patient to walk, to have independency in his/ her activities of daily living, with good faith in social participation and acceptance. Management of arthrogryposis in a PMR department is indicated at all stages, including the perioperative period and in case of complications.

Contributors: LDS, AJS: conceptualization, data curation, formal analysis, investigation, methodology, project administration, validation, visualization, writing - original draft; LNS was responsible for investigation, methodology, validation, visualization, writing - review \& editing.. LDS will act as a study guarantor. All authors approved the final version of this manuscript and are responsible for all aspects of this study.

Funding: None; Competing interests: None stated.

\section{References}

1. Hall JG. Arthrogryposis multiplex congenita: etiology, genetics, classification, diagnostic approach and general aspects. J Pediatr Orthop B. 1997;6:159-166.

2. Hall J. Arthrogryposis. In: Cassidy S, Allanson J, editors. Management of genetic syndromes, 2nd ed. Hoboken (NJ): Wiley \& Sons. 2005:63-75.

3. Hall JG. Arthrogryposis (multiple congenital contractures): diagnostic approach to etiology, classification, genetics, and general principles. Eur J Med Genet. 2014;57:464-472.

4. Polizzi A, Huson SM, Vincent A. Teratogen update: maternal myasthenia gravis as a cause of congenital arthrogryposis. Teratology. 2000;62:332-341.

5. Fuller DJ. Immobilization of fetal joints: a cause of progressive prenatal deformity. J Bone Joint Surg Br. 1975;57:115.

6. Drachman DB, Coulombre AJ. Experimental clubfoot and arthrogryposis multiplex congenita. Lancet. 1962;2:523-526.

7. Robertson GG, Williamson AP, Blattner RJ. A study of abnormalities in early chick embryos inoculated with New Castle virus. J Exp Zool. 1955;129:5.

8. Swinyard CA, Bleck EE. The etiology of arthrogryposis. Clin Orthop. 1985;194:15-29.

9. Chen H. Atlas of Genetic Diagnosis and Counseling. Springer-Verlag New York. 2006.

10. Binkiewicz-Glinska A, Sobierajska-Rek A, Bakula S, Wierzba J, Drewek K, Kowalski I, et al. Arthrogryposis in infancy, multidisciplinary approach: case report. BMC Pediatrics. 2013;13.

11. Kimber E, Tajsharghi H, Kroksmark AK, Oldfors A, Tulinius M. Distal arthrogryposis: clinical and genetic 
findings. Acta Paediatrica. 2012;101:877887.

12. Sarwark JF, MacEwen GD, Scott CI. Jr. Amyoplasia (a common form of arthrogryposis). J Bone Joint Surg. 1990;72:465-469.

13. O'Flaherty P. Arthrogryposis multiplex congenita. Neonatal Netw. 2001;20:13-20.

14. Moessinger AC. Fetal akinesia deformation sequence: an animal model. Pediatrics. 1983;72:857-863.

15. Hall JG, Opitz JM, Reynolds JF. Analysis of Pena Shokeir phenotype. Am J Med Genet. 1986;25:99-117.
16. Piper SL, Dicke JM, Wall LB, Shen TS, Goldfarb CA. Prenatal detection of upper limb differences with obstetric ultrasound. J Hand Surg Am. 2015;40:13101317.

17. Azbell K, Dannemiller L. A case report of an infant with arthrogryposis. Pediatr Phys Ther. 2015;27:293-301.

18. Herring JA. Tachdjian's Pediatric Orthopaedics. WB Saunders Company, Philadelphia, Pa, USA, $3^{\text {rd }}$ edition, 2002. 